Data Repository: Time scales of pluton construction at differing crustal levels: Examples from the Mount Stuart and Tenpeak intrusions, North Cascades, Washington

Jennifer E.P. Matzel, Samuel A. Bowring, Robert B. Miller

\title{
References Cited
}

Jaffey, A.H., Flynn, K.F., Glendenin, L.E., Bentley, W.C., and Essling, A.M., 1971, Precision measurements of half-lives and specific activities of ${ }^{235} \mathrm{U}$ and ${ }^{238} \mathrm{U}$ : Physical Review, v. C4, p. 1889-1906.

Ludwig, K.R., 1980, Calculation of uncertainties of U-Pb isotope data: Earth and Planetary Science Letters, v. 46, p. 212-220, doi: 10.1016/0012-821X(80)90007-2. 
Table DR1. U-Pb analyses from the Mount Stuart batholith.

\begin{tabular}{|c|c|c|c|c|c|c|c|c|c|c|c|c|c|c|c|c|c|c|c|c|c|c|}
\hline \multirow[b]{2}{*}{$\begin{array}{l}\text { Frac } \\
\text { (a) }\end{array}$} & \multicolumn{6}{|c|}{ Composition } & \multicolumn{9}{|c|}{ Isotopic Ratios } & \multicolumn{6}{|c|}{ Dates (Ma) } & \multirow[b]{2}{*}{$\begin{array}{l}\text { Discord- } \\
\text { ance } \\
\text { (j) } \\
\end{array}$} \\
\hline & $\begin{array}{c}\mathrm{Wt} \\
(\mu \mathrm{g}) \\
(\mathrm{b}) \\
\end{array}$ & $\begin{array}{c}\mathrm{U} \\
(\mathrm{ppm})\end{array}$ & $\begin{array}{c}\mathrm{Pb} \\
(\mathrm{ppm})\end{array}$ & $\begin{array}{l}\underline{T h} \\
\mathrm{U} \\
\text { (c) }\end{array}$ & $\begin{array}{l}\frac{\mathrm{Pb} *}{\mathrm{Pbc}} \\
\text { (d) } \\
\end{array}$ & $\begin{array}{l}\mathrm{Pbc} \\
\text { (pg) } \\
\text { (d) } \\
\end{array}$ & $\begin{array}{c}\frac{{ }^{206} \mathrm{~Pb}}{{ }^{204} \mathrm{~Pb}} \\
(\mathrm{e}) \\
\end{array}$ & $\begin{array}{c}{ }^{208} \mathrm{~Pb} \\
(\mathrm{fb}) \\
\end{array}$ & $\begin{array}{c}\frac{206 \mathrm{~Pb}}{{ }^{238} \mathrm{U}} \\
\text { (f) } \\
\end{array}$ & $\begin{array}{c}\% \text { err } \\
\text { (g) } \\
\end{array}$ & $\begin{array}{c}\frac{{ }^{207} \mathrm{~Pb}}{{ }^{235} \mathrm{U}} \\
\text { (f) }\end{array}$ & $\begin{array}{c}\% \text { err } \\
\text { (g) } \\
\end{array}$ & $\begin{array}{c}{ }^{20 /} \mathrm{Pb} \\
(\mathrm{f}) \\
\end{array}$ & $\begin{array}{c}\% \text { err } \\
\text { (g) }\end{array}$ & $\begin{array}{l}\text { corr. } \\
\text { coef. }\end{array}$ & $\begin{array}{c}{ }^{206} \mathrm{~Pb} \\
{ }^{238} \mathrm{U} \\
(\mathrm{h}) \\
\end{array}$ & $\begin{array}{l} \pm \\
\text { (i) } \\
\end{array}$ & $\begin{array}{l}\frac{{ }^{20 /} \mathrm{Pb}}{{ }^{235} \mathrm{U}} \\
\text { (h) } \\
\end{array}$ & $\begin{array}{l} \pm \\
\text { (i) } \\
\end{array}$ & $\begin{array}{c}\frac{{ }^{207} \mathrm{~Pb}}{{ }^{206} \mathrm{~Pb}} \\
\text { (h) }\end{array}$ & $\begin{array}{l} \pm \\
\text { (i) } \\
\end{array}$ & \\
\hline \multicolumn{23}{|c|}{ MS2: GABBRO } \\
\hline $\mathrm{z} 2$ & 88.6 & 73.1 & 1.0 & 0.347 & 59.6 & 1.5 & 3794 & 0.111 & 0.014187 & 0.10 & 0.09329 & 0.27 & 0.04769 & 0.24 & 0.439 & 90.81 & 0.09 & 90.56 & 0.26 & 84.1 & 5.8 & -8.0 \\
\hline z3 & 64.1 & 79.2 & 1.1 & 0.296 & 117 & 0.6 & 7531 & 0.095 & 0.014179 & 0.05 & 0.09350 & 0.12 & 0.04782 & 0.11 & 0.457 & 90.76 & 0.05 & 90.76 & 0.11 & 90.5 & 2.5 & -0.3 \\
\hline $\mathrm{z} 4$ & 38.6 & 68.2 & 1.0 & 0.349 & 48.0 & 0.8 & 3057 & 0.112 & 0.014192 & 0.08 & 0.09364 & 0.13 & 0.04785 & 0.10 & 0.655 & 90.85 & 0.07 & 90.89 & 0.12 & 92.1 & 2.3 & 1.4 \\
\hline z5 & 93.6 & 42.0 & 0.6 & 0.333 & 45.4 & 1.2 & 2905 & 0.107 & 0.014187 & 0.08 & 0.09362 & 0.12 & 0.04786 & 0.09 & 0.693 & 90.81 & 0.07 & 90.87 & 0.12 & 92.3 & 2.1 & 1.7 \\
\hline z6 & 49.3 & 76.4 & 1.1 & 0.318 & 25.2 & 2.1 & 1629 & 0.102 & 0.014184 & 0.12 & 0.09364 & 0.19 & 0.04788 & 0.14 & 0.672 & 90.80 & 0.11 & 90.89 & 0.18 & 93.4 & 3.3 & 2.8 \\
\hline z7 & 93.6 & 55.8 & 0.8 & 0.335 & 16.0 & 4.6 & 1036 & 0.107 & 0.014173 & 0.28 & 0.09358 & 0.39 & 0.04789 & 0.26 & 0.741 & 90.73 & 0.25 & 90.84 & 0.37 & 93.7 & 6.2 & 3.2 \\
\hline z8 & 47.1 & 83.5 & 1.1 & 0.252 & 27.3 & 2.0 & 1795 & 0.081 & 0.014167 & 0.13 & 0.09339 & 0.22 & 0.04781 & 0.18 & 0.614 & 90.69 & 0.11 & 90.66 & 0.21 & 89.9 & 4.2 & -0.9 \\
\hline z9 & 54.2 & 170.8 & 2.3 & 0.242 & 65.2 & 1.9 & 4265 & 0.078 & 0.014135 & 0.06 & 0.09321 & 0.11 & 0.04783 & 0.09 & 0.585 & 90.48 & 0.06 & 90.49 & 0.11 & 90.8 & 2.2 & 0.3 \\
\hline \multicolumn{23}{|c|}{ MS4: GABBRO } \\
\hline $\mathrm{z} 2$ & 25.0 & 162.3 & 2.3 & 0.381 & 44.5 & 1.3 & 2807 & 0.122 & 0.014196 & 0.08 & 0.09357 & 0.14 & 0.04780 & 0.12 & 0.592 & 90.87 & 0.07 & 90.82 & 0.14 & 89.6 & 2.8 & -1.5 \\
\hline z3 & 49.0 & 62.5 & 0.9 & 0.362 & 29.3 & 1.5 & 1867 & 0.116 & 0.014218 & 0.15 & 0.09388 & 0.40 & 0.04789 & 0.35 & 0.489 & 91.01 & 0.14 & 91.12 & 0.38 & 93.8 & 8.3 & 3.0 \\
\hline z6 & 27.2 & 49.8 & 0.7 & 0.358 & 3.9 & 5.0 & 263 & 0.115 & 0.014219 & 0.75 & 0.09432 & 0.91 & 0.04811 & 0.50 & 0.838 & 91.02 & 0.68 & 91.52 & 0.87 & 104.6 & 11.7 & 13.0 \\
\hline z7 & 38.3 & 51.9 & 0.7 & 0.384 & 6.7 & 4.2 & 440 & 0.123 & 0.014209 & 0.43 & 0.09400 & 0.55 & 0.04798 & 0.33 & 0.806 & 90.95 & 0.39 & 91.22 & 0.53 & 98.2 & 7.8 & 7.4 \\
\hline z11 & 46.6 & 37.9 & 0.5 & 0.345 & 17.2 & 1.5 & 1106 & 0.111 & 0.014212 & 0.17 & 0.09424 & 0.33 & 0.04809 & 0.28 & 0.541 & 90.97 & 0.16 & 91.45 & 0.32 & 103.9 & 6.6 & 12.4 \\
\hline $\mathrm{z} 12$ & 44.8 & 42.6 & 0.6 & 0.379 & 19.9 & 1.4 & 1266 & 0.121 & 0.014208 & 0.15 & 0.09386 & 0.22 & 0.04791 & 0.16 & 0.708 & 90.95 & 0.14 & 91.09 & 0.21 & 94.8 & 3.7 & 4.1 \\
\hline z13 & 34.7 & 43.5 & 0.6 & 0.387 & 4.6 & 4.7 & 303 & 0.125 & 0.014206 & 0.64 & 0.09410 & 0.81 & 0.04804 & 0.47 & 0.814 & 90.93 & 0.58 & 91.31 & 0.77 & 101.3 & 11.1 & 10.2 \\
\hline \multicolumn{23}{|c|}{ MS5:TONALITE } \\
\hline $\mathrm{z} 1$ & 39.2 & 820.4 & 11.8 & 0.202 & 121 & 3.8 & 7986 & 0.065 & 0.014987 & 0.06 & 0.09917 & 0.08 & 0.04799 & 0.06 & 0.662 & 95.89 & 0.05 & 96.01 & 0.08 & 98.8 & 1.5 & 2.9 \\
\hline z2 & 34.6 & 1007 & 14.4 & 0.182 & 333 & 1.5 & 22123 & 0.058 & 0.014996 & 0.07 & 0.09917 & 0.09 & 0.04797 & 0.06 & 0.765 & 95.95 & 0.06 & 96.01 & 0.08 & 97.6 & 1.3 & 1.7 \\
\hline z3 & 30.6 & 1171 & 16.6 & 0.167 & 239 & 2.1 & 15947 & 0.053 & 0.014996 & 0.06 & 0.09914 & 0.09 & 0.04795 & 0.06 & 0.731 & 95.95 & 0.06 & 95.98 & 0.08 & 96.6 & 1.4 & 0.7 \\
\hline $\mathrm{z} 5 \mathrm{a}$ & 18.5 & 698.9 & 9.8 & 0.145 & 137 & 1.3 & 9219 & 0.046 & 0.014984 & 0.05 & 0.09901 & 0.08 & 0.04792 & 0.06 & 0.657 & 95.88 & 0.05 & 95.86 & 0.08 & 95.4 & 1.5 & -0.5 \\
\hline z5b & 21.9 & 687.0 & 9.6 & 0.119 & 174 & 1.2 & 11786 & 0.038 & 0.014980 & 0.05 & 0.09912 & 0.08 & 0.04799 & 0.07 & 0.541 & 95.85 & 0.04 & 95.96 & 0.08 & 98.7 & 1.7 & 2.9 \\
\hline \multicolumn{23}{|c|}{ MS6:GRANODIORITE } \\
\hline $\mathrm{z} 1$ & 22.0 & 1061 & 15.0 & 0.162 & 134 & 2.5 & 8959 & 0.052 & 0.014977 & 0.06 & 0.09914 & 0.08 & 0.04801 & 0.05 & 0.742 & 95.83 & 0.06 & 95.98 & 0.08 & 99.8 & 1.3 & 4.0 \\
\hline z2 & 18.0 & 1162 & 16.2 & 0.118 & 135 & 2.2 & 9175 & 0.038 & 0.014969 & 0.07 & 0.09902 & 0.10 & 0.04798 & 0.06 & 0.749 & 95.78 & 0.07 & 95.87 & 0.09 & 98.2 & 1.5 & 2.5 \\
\hline z3 & 25.0 & 1232 & 17.3 & 0.146 & 488 & 0.9 & 32770 & 0.047 & 0.014953 & 0.11 & 0.09896 & 0.13 & 0.04800 & 0.06 & 0.898 & 95.68 & 0.11 & 95.82 & 0.12 & 99.3 & 1.3 & 3.6 \\
\hline z5 & 20.2 & 822 & 11.6 & 0.148 & 104 & 2.3 & 6958 & 0.047 & 0.014976 & 0.09 & 0.09910 & 0.16 & 0.04799 & 0.13 & 0.578 & 95.82 & 0.09 & 95.94 & 0.16 & 99.0 & 3.2 & 3.2 \\
\hline z6 & 10.3 & 1830 & 25.6 & 0.124 & 25 & 10 & 1718 & 0.040 & 0.014959 & 0.21 & 0.09906 & 0.27 & 0.04803 & 0.17 & 0.791 & 95.72 & 0.20 & 95.91 & 0.26 & 100.7 & 3.9 & 4.9 \\
\hline z7 & 19.1 & 198 & 2.8 & 0.198 & 16 & 3.5 & 1045 & 0.063 & 0.014983 & 0.18 & 0.09917 & 0.27 & 0.04800 & 0.19 & 0.714 & 95.87 & 0.17 & 96.01 & 0.25 & 99.5 & 4.4 & 3.6 \\
\hline z9a & 10.4 & 1131 & 15.9 & 0.150 & 171 & 1.0 & 11434 & 0.048 & 0.014915 & 0.05 & 0.09864 & 0.08 & 0.04796 & 0.06 & 0.653 & 95.44 & 0.05 & 95.52 & 0.08 & 97.5 & 1.5 & 2.1 \\
\hline z10a & 13.7 & 1028 & 14.5 & 0.146 & 185 & 1.1 & 12453 & 0.047 & 0.014963 & 0.05 & 0.09883 & 0.09 & 0.04791 & 0.08 & 0.585 & 95.74 & 0.05 & 95.70 & 0.09 & 94.6 & 1.8 & -1.2 \\
\hline $\mathrm{z} 10 \mathrm{~b}$ & 16.7 & 1286 & 18.4 & 0.157 & 260 & 1.2 & 17389 & 0.051 & 0.015149 & 0.05 & 0.10077 & 0.07 & 0.04824 & 0.06 & 0.638 & 96.92 & 0.04 & 97.49 & 0.07 & 111.3 & 1.3 & 12.9 \\
\hline $\mathrm{z} 10 \mathrm{c}$ & 11.4 & 836 & 11.7 & 0.131 & 111 & 1.2 & 7522 & 0.042 & 0.014949 & 0.05 & 0.09882 & 0.09 & 0.04795 & 0.08 & 0.542 & 95.65 & 0.05 & 95.69 & 0.08 & 96.6 & 1.8 & 1.0 \\
\hline \multicolumn{23}{|c|}{ MS13:GRANODIORITE } \\
\hline $\mathrm{z} 1$ & 11.8 & 235.0 & 3.3 & 0.345 & 12.4 & 3.1 & 806 & 0.110 & 0.014177 & 0.24 & 0.09358 & 0.47 & 0.04787 & 0.39 & 0.573 & 90.75 & 0.22 & 90.84 & 0.45 & 93.1 & 9.2 & 2.5 \\
\hline z2 & 18.7 & 99.6 & 1.4 & 0.312 & 15.9 & 1.6 & 1037 & 0.100 & 0.014169 & 0.19 & 0.09375 & 0.30 & 0.04799 & 0.22 & 0.662 & 90.70 & 0.17 & 90.99 & 0.28 & 98.8 & 5.3 & 8.2 \\
\hline z3 & 5.5 & 84.7 & 1.2 & 0.406 & 4.8 & 1.4 & 315 & 0.130 & 0.014219 & 0.61 & 0.09404 & 1.22 & 0.04797 & 1.01 & 0.568 & 91.02 & 0.55 & 91.26 & 1.16 & 97.6 & 23.8 & 6.7 \\
\hline $\mathrm{z} 4$ & 19.6 & 143.9 & 2.0 & 0.355 & 13.6 & 2.9 & 875 & 0.114 & 0.014207 & 0.22 & 0.09387 & 0.30 & 0.04792 & 0.19 & 0.776 & 90.94 & 0.20 & 91.10 & 0.28 & 95.3 & 4.4 & 4.6 \\
\hline
\end{tabular}


Table DR1 (cont.). U-Pb analyses from the Mount Stuart batholith.

\begin{tabular}{|c|c|c|c|c|c|c|c|c|c|c|c|c|c|c|c|c|c|c|c|c|c|c|}
\hline \multirow[b]{2}{*}{$\begin{array}{l}\text { Frac } \\
\text { (a) }\end{array}$} & \multicolumn{6}{|c|}{ Composition } & \multicolumn{9}{|c|}{ Isotopic Ratios } & \multicolumn{6}{|c|}{ Dates (Ma) } & \multirow[b]{2}{*}{$\begin{array}{l}\text { Discord- } \\
\text { ance } \\
\text { (j) } \\
\end{array}$} \\
\hline & $\begin{array}{c}\mathrm{Wt} \\
(\mu \mathrm{g}) \\
\text { (b) } \\
\end{array}$ & $\begin{array}{c}\mathrm{U} \\
(\mathrm{ppm})\end{array}$ & $\begin{array}{c}\mathrm{Pb} \\
(\mathrm{ppm})\end{array}$ & $\begin{array}{c}\frac{\mathrm{Th}}{\mathrm{U}} \\
\text { (c) } \\
\end{array}$ & $\begin{array}{l}\frac{\mathrm{Pb}^{*}}{\mathrm{Pbc}} \\
\text { (d) } \\
\end{array}$ & $\begin{array}{l}\mathrm{Pbc} \\
\text { (pg) } \\
\text { (d) } \\
\end{array}$ & $\begin{array}{c}\frac{{ }^{206} \mathrm{~Pb}}{{ }^{204} \mathrm{~Pb}} \\
(\mathrm{e}) \\
\end{array}$ & $\begin{array}{l}\frac{{ }^{208} \mathrm{~Pb}}{206 \mathrm{~Pb}} \\
\text { (f) } \\
\end{array}$ & $\begin{array}{c}\frac{{ }^{206} \mathrm{~Pb}}{{ }^{238} \mathrm{U}} \\
(\mathrm{f}) \\
\end{array}$ & $\begin{array}{c}\% \text { err } \\
\text { (g) } \\
\end{array}$ & $\begin{array}{c}\frac{{ }^{20 /} \mathrm{Pb}}{{ }^{235} \mathrm{U}} \\
(\mathrm{f}) \\
\end{array}$ & $\begin{array}{c}\text { \% err } \\
\text { (g) } \\
\end{array}$ & $\begin{array}{c}\frac{207}{206} \mathrm{~Pb} \\
(\mathrm{f}) \\
\end{array}$ & $\begin{array}{c}\% \text { err } \\
(\mathrm{g}) \\
\end{array}$ & $\begin{array}{l}\text { corr. } \\
\text { coef. }\end{array}$ & $\begin{array}{c}\frac{{ }^{206} \mathrm{~Pb}}{{ }^{238} \mathrm{U}} \\
(\mathrm{h}) \\
\end{array}$ & $\begin{array}{c} \pm \\
\text { (i) } \\
\end{array}$ & $\begin{array}{c}\frac{{ }^{200} \mathrm{~Pb}}{{ }^{235} \mathrm{U}} \\
(\mathrm{h}) \\
\end{array}$ & $\begin{array}{c} \pm \\
\text { (i) } \\
\end{array}$ & $\begin{array}{l}\frac{{ }^{207} \mathrm{~Pb}}{206 \mathrm{~Pb}} \\
\text { (h) } \\
\end{array}$ & $\begin{array}{c} \pm \\
\text { (i) } \\
\end{array}$ & \\
\hline \multicolumn{23}{|c|}{ MS13:GRANODIORITE (cont.) } \\
\hline z5 & 7.2 & 135.5 & 1.9 & 0.329 & 14.4 & 0.9 & 933 & 0.105 & 0.014176 & 0.20 & 0.09342 & 0.49 & 0.04780 & 0.42 & 0.505 & 90.74 & 0.18 & 90.68 & 0.46 & 89.2 & 10.0 & -1.7 \\
\hline z6 & 10.7 & 229.4 & 3.5 & 0.614 & 14.5 & 2.6 & 871 & 0.196 & 0.014200 & 0.23 & 0.09349 & 0.29 & 0.04775 & 0.18 & 0.783 & 90.89 & 0.20 & 90.75 & 0.28 & 87.0 & 4.3 & -4.5 \\
\hline $\mathrm{z} 7$ & 5.9 & 246.5 & 3.5 & 0.315 & 14.5 & 1.4 & 945 & 0.101 & 0.014168 & 0.20 & 0.09365 & 0.27 & 0.04794 & 0.17 & 0.769 & 90.69 & 0.18 & 90.90 & 0.25 & 96.5 & 4.0 & 6.0 \\
\hline \multicolumn{23}{|c|}{ MS17C:DIORITE OF THE BIG JIM COMPLEX } \\
\hline $\mathrm{z} 1$ & 38.2 & 284.9 & 4.4 & 0.493 & 135 & 1.3 & 8244 & 0.158 & 0.014980 & 0.06 & 0.09904 & 0.09 & 0.04795 & 0.07 & 0.655 & 95.85 & 0.05 & 95.89 & 0.08 & 96.7 & 1.5 & 0.9 \\
\hline $\mathrm{z} 2$ & 30.8 & 214.5 & 3.3 & 0.472 & 14.7 & 6.9 & 919 & 0.151 & 0.014989 & 0.21 & 0.09923 & 0.28 & 0.04801 & 0.18 & 0.772 & 95.91 & 0.20 & 96.07 & 0.26 & 100.0 & 4.2 & 4.1 \\
\hline z3 & 28.0 & 157.0 & 2.4 & 0.457 & 48.7 & 1.4 & 3007 & 0.147 & 0.014940 & 0.08 & 0.09896 & 0.16 & 0.04804 & 0.13 & 0.576 & 95.60 & 0.08 & 95.81 & 0.15 & 101.1 & 3.0 & 5.4 \\
\hline $\mathrm{z} 4$ & 26.2 & 219.6 & 3.4 & 0.454 & 73.7 & 1.2 & 4550 & 0.145 & 0.014991 & 0.06 & 0.09922 & 0.11 & 0.04800 & 0.09 & 0.583 & 95.92 & 0.06 & 96.05 & 0.10 & 99.3 & 2.1 & 3.4 \\
\hline z5 & 41.8 & 185.5 & 2.9 & 0.474 & 34.3 & 3.5 & 2115 & 0.152 & 0.014981 & 0.10 & 0.09905 & 0.15 & 0.04795 & 0.11 & 0.673 & 95.86 & 0.09 & 95.90 & 0.15 & 97.0 & 2.7 & 1.2 \\
\hline z6 & 60.7 & 254.5 & 3.9 & 0.440 & 31.1 & 7.6 & 1934 & 0.141 & 0.014973 & 0.11 & 0.09891 & 0.15 & 0.04791 & 0.10 & 0.723 & 95.81 & 0.10 & 95.77 & 0.14 & 94.9 & 2.5 & -0.9 \\
\hline z7 & 35.4 & 189.0 & 3.0 & 0.516 & 67.3 & 1.5 & 4089 & 0.165 & 0.014981 & 0.07 & 0.09899 & 0.13 & 0.04793 & 0.10 & 0.563 & 95.86 & 0.06 & 95.85 & 0.12 & 95.6 & 2.5 & -0.3 \\
\hline z8 & 23.6 & 274.5 & 4.3 & 0.482 & 23.9 & 4.2 & 1473 & 0.155 & 0.014986 & 0.14 & 0.09926 & 0.25 & 0.04804 & 0.20 & 0.599 & 95.89 & 0.13 & 96.09 & 0.24 & 101.0 & 4.8 & 5.1 \\
\hline z9 & 35.9 & 338.7 & 5.3 & 0.496 & 42.3 & 4.5 & 2591 & 0.159 & 0.014992 & 0.11 & 0.09906 & 0.18 & 0.04792 & 0.14 & 0.605 & 95.93 & 0.10 & 95.91 & 0.17 & 95.3 & 3.4 & -0.6 \\
\hline z10 & 36.1 & 302.9 & 4.7 & 0.516 & 69.3 & 2.5 & 4208 & 0.165 & 0.014978 & 0.06 & 0.09886 & 0.11 & 0.04787 & 0.09 & 0.594 & 95.84 & 0.06 & 95.72 & 0.10 & 92.9 & 2.0 & -3.2 \\
\hline z11 & 26.7 & 245.7 & 3.8 & 0.488 & 49.2 & 2.1 & 3015 & 0.156 & 0.014996 & 0.07 & 0.09912 & 0.12 & 0.04794 & 0.09 & 0.665 & 95.95 & 0.07 & 95.96 & 0.11 & 96.3 & 2.1 & 0.4 \\
\hline \multicolumn{23}{|c|}{ MS24:TONALITE } \\
\hline $\mathrm{z} 1$ & 44.3 & 99.8 & 1.5 & 0.379 & 51.4 & 1.3 & 3244 & 0.121 & 0.014478 & 0.10 & 0.09561 & 0.23 & 0.04790 & 0.20 & 0.492 & 92.67 & 0.09 & 92.72 & 0.22 & 94.1 & 4.7 & 1.5 \\
\hline z2 & 11.4 & 190.6 & 2.7 & 0.245 & 9.8 & 3.1 & 656 & 0.079 & 0.014495 & 0.31 & 0.09590 & 0.80 & 0.04798 & 0.71 & 0.480 & 92.77 & 0.29 & 92.98 & 0.76 & 98.5 & 16.7 & 5.8 \\
\hline z3 & 13.7 & 138.9 & 1.9 & 0.218 & 7.5 & 3.5 & 511 & 0.070 & 0.014475 & 0.38 & 0.09577 & 0.56 & 0.04798 & 0.40 & 0.710 & 92.64 & 0.35 & 92.86 & 0.54 & 98.5 & 9.4 & 5.9 \\
\hline $\mathrm{z} 4$ & 47.3 & 121.5 & 1.7 & 0.251 & 19.7 & 4.1 & 1302 & 0.080 & 0.014476 & 0.16 & 0.09566 & 0.28 & 0.04792 & 0.23 & 0.605 & 92.65 & 0.15 & 92.76 & 0.27 & 95.5 & 5.3 & 3.0 \\
\hline z5 & 6.3 & 194.0 & 2.7 & 0.268 & 25.3 & 0.7 & 1654 & 0.086 & 0.014479 & 0.12 & 0.09562 & 0.20 & 0.04790 & 0.17 & 0.584 & 92.67 & 0.11 & 92.73 & 0.20 & 94.1 & 3.9 & 1.5 \\
\hline z9 & 5.0 & 214.9 & 3.0 & 0.225 & 5.8 & 2.6 & 396 & 0.072 & 0.014473 & 0.50 & 0.09586 & 0.96 & 0.04804 & 0.78 & 0.589 & 92.63 & 0.46 & 92.95 & 0.92 & 101.2 & 18.4 & 8.4 \\
\hline \multicolumn{23}{|c|}{ MS26:TONALITE } \\
\hline $\mathrm{z} 1$ & 22.0 & 310.7 & 4.4 & 0.175 & 44.2 & 2.2 & 2956 & 0.056 & 0.015037 & 0.08 & 0.09944 & 0.11 & 0.04796 & 0.08 & 0.693 & 96.21 & 0.07 & 96.26 & 0.11 & 97.4 & 1.9 & 1.2 \\
\hline z2 & 10.9 & 182.9 & 2.6 & 0.169 & 16.2 & 1.7 & 1097 & 0.054 & 0.015044 & 0.18 & 0.09959 & 0.24 & 0.04801 & 0.16 & 0.757 & 96.26 & 0.17 & 96.40 & 0.23 & 99.9 & 3.7 & 3.6 \\
\hline z3 & 9.1 & 189.9 & 2.7 & 0.176 & 7.9 & 3.1 & 541 & 0.056 & 0.015048 & 0.35 & 0.09979 & 0.49 & 0.04809 & 0.33 & 0.744 & 96.28 & 0.34 & 96.58 & 0.47 & 103.9 & 7.8 & 7.3 \\
\hline $\mathrm{z} 4$ & 8.6 & 148.3 & 2.1 & 0.160 & 23.1 & 0.8 & 1562 & 0.051 & 0.015067 & 0.13 & 0.09970 & 0.22 & 0.04799 & 0.17 & 0.613 & 96.40 & 0.12 & 96.50 & 0.21 & 98.8 & 4.0 & 2.5 \\
\hline z5 & 7.9 & 80.0 & 1.2 & 0.193 & 13.0 & 0.7 & 878 & 0.062 & 0.015048 & 0.23 & 0.09961 & 0.90 & 0.04801 & 0.83 & 0.444 & 96.28 & 0.22 & 96.41 & 0.86 & 99.6 & 19.5 & 3.4 \\
\hline \multicolumn{23}{|c|}{ MS31:TONALITE } \\
\hline $\mathrm{z} 1$ & 56.8 & 95.0 & 1.3 & 0.307 & 29.6 & 2.5 & 1910 & 0.098 & 0.014192 & 0.12 & 0.09363 & 0.23 & 0.04785 & 0.19 & 0.562 & 90.84 & 0.11 & 90.88 & 0.22 & 91.9 & 4.5 & 1.2 \\
\hline z2 & 78.8 & 220.2 & 3.0 & 0.169 & 36.7 & 6.3 & 2460 & 0.054 & 0.014176 & 0.11 & 0.09364 & 0.16 & 0.04791 & 0.11 & 0.720 & 90.75 & 0.10 & 90.89 & 0.14 & 94.7 & 2.6 & 4.2 \\
\hline $\mathrm{z} 4$ & 33.3 & 88.9 & 1.3 & 0.362 & 56.5 & 0.7 & 3581 & 0.116 & 0.014181 & 0.13 & 0.09352 & 0.25 & 0.04783 & 0.21 & 0.546 & 90.77 & 0.12 & 90.78 & 0.24 & 90.8 & 5.0 & 0.1 \\
\hline z5 & 65.2 & 85.0 & 1.2 & 0.343 & 21.1 & 3.7 & 1356 & 0.110 & 0.014193 & 0.14 & 0.09365 & 0.19 & 0.04785 & 0.12 & 0.783 & 90.85 & 0.13 & 90.90 & 0.18 & 92.1 & 2.7 & 1.3 \\
\hline z6 & 75.3 & 145.9 & 2.0 & 0.180 & 145 & 1.0 & 9646 & 0.058 & 0.014173 & 0.07 & 0.09348 & 0.09 & 0.04784 & 0.06 & 0.737 & 90.72 & 0.06 & 90.74 & 0.08 & 91.1 & 1.5 & 0.5 \\
\hline $\mathrm{z} 7$ & 37.8 & 42.9 & 0.6 & 0.404 & 25.8 & 0.9 & 1626 & 0.130 & 0.014240 & 0.12 & 0.09434 & 0.25 & 0.04805 & 0.21 & 0.564 & 91.15 & 0.11 & 91.53 & 0.24 & 101.6 & 4.9 & 10.3 \\
\hline \multicolumn{23}{|c|}{ MS37:TONALITE } \\
\hline $\mathrm{z} 1$ & 5.8 & 322.0 & 4.4 & 0.171 & 13.9 & 1.8 & 945 & 0.055 & 0.014470 & 0.20 & 0.09561 & 0.33 & 0.04792 & 0.25 & 0.648 & 92.61 & 0.18 & 92.72 & 0.32 & 95.4 & 6.0 & 2.9 \\
\hline z2 & 1.9 & 700.7 & 9.6 & 0.163 & 6.9 & 2.6 & 480 & 0.052 & 0.014466 & 0.40 & 0.09584 & 0.59 & 0.04805 & 0.42 & 0.709 & 92.59 & 0.36 & 92.92 & 0.56 & 101.6 & 9.9 & 8.8 \\
\hline
\end{tabular}


Table DR1 (cont.). U-Pb analyses from the Mount Stuart batholith.

\begin{tabular}{|c|c|c|c|c|c|c|c|c|c|c|c|c|c|c|c|c|c|c|c|c|c|c|}
\hline \multirow[b]{2}{*}{$\begin{array}{l}\text { Frac } \\
\text { (a) }\end{array}$} & \multicolumn{6}{|c|}{ Composition } & \multicolumn{9}{|c|}{ Isotopic Ratios } & \multicolumn{6}{|c|}{ Dates (Ma) } & \multirow[b]{2}{*}{$\begin{array}{l}\text { Discord- } \\
\text { ance } \\
\text { (j) } \\
\end{array}$} \\
\hline & $\begin{array}{c}\text { Wt } \\
(\mu g) \\
(b) \\
\end{array}$ & $\begin{array}{c}\mathrm{U} \\
(\mathrm{ppm})\end{array}$ & $\begin{array}{c}\mathrm{Pb} \\
(\mathrm{ppm})\end{array}$ & $\begin{array}{c}\frac{T h}{U} \\
\text { (c) } \\
\end{array}$ & $\begin{array}{l}\frac{\mathrm{Pb}^{*}}{\mathrm{Pbc}} \\
\text { (d) } \\
\end{array}$ & $\begin{array}{l}\mathrm{Pbc} \\
\text { (pg) } \\
\text { (d) } \\
\end{array}$ & $\begin{array}{c}\frac{{ }^{206} \mathrm{~Pb}}{{ }^{204} \mathrm{~Pb}} \\
(\mathrm{e}) \\
\end{array}$ & $\begin{array}{c}\frac{{ }^{208} \mathrm{~Pb}}{{ }^{206} \mathrm{~Pb}} \\
\text { (f) } \\
\end{array}$ & $\begin{array}{l}\frac{{ }^{206} \mathrm{~Pb}}{{ }^{238} \mathrm{U}} \\
(\mathrm{f}) \\
\end{array}$ & $\begin{array}{c}\% \text { err } \\
\text { (g) } \\
\end{array}$ & $\begin{array}{c}\frac{{ }^{207} \mathrm{~Pb}}{{ }^{235} \mathrm{U}} \\
\text { (f) } \\
\end{array}$ & $\begin{array}{c}\text { \% err } \\
\text { (g) } \\
\end{array}$ & $\begin{array}{c}{ }^{207} \mathrm{~Pb} \\
{ }^{206} \mathrm{~Pb} \\
(\mathrm{f}) \\
\end{array}$ & $\begin{array}{c}\text { \% err } \\
\text { (g) } \\
\end{array}$ & $\begin{array}{l}\text { corr. } \\
\text { coef. }\end{array}$ & $\begin{array}{c}\frac{{ }^{206} \mathrm{~Pb}}{{ }^{238} \mathrm{U}} \\
(\mathrm{h}) \\
\end{array}$ & $\begin{array}{r} \pm \\
\text { (i) } \\
\end{array}$ & $\begin{array}{l}\frac{{ }^{20 /} \mathrm{Pb}}{{ }^{235} \mathrm{U}} \\
(\mathrm{h}) \\
\end{array}$ & $\begin{array}{l} \pm \\
\text { (i) } \\
\end{array}$ & $\begin{array}{c}\frac{{ }^{207} \mathrm{~Pb}}{206 \mathrm{~Pb}} \\
(\mathrm{~h}) \\
\end{array}$ & $\begin{array}{l} \pm \\
\text { (i) } \\
\end{array}$ & \\
\hline \multicolumn{23}{|c|}{ MS37:TONALITE (cont.) } \\
\hline z3 & 2.7 & 301.1 & 4.2 & 0.261 & 5.2 & 2.2 & 352 & 0.084 & 0.014475 & 0.55 & 0.09602 & 1.12 & 0.04811 & 0.93 & 0.567 & 92.64 & 0.50 & 93.10 & 1.07 & 104.8 & 21.9 & 11.6 \\
\hline $\mathrm{z} 4$ & 1.5 & 506.3 & 6.9 & 0.145 & 2.8 & 3.6 & 208 & 0.047 & 0.014522 & 0.96 & 0.09665 & 1.26 & 0.04827 & 0.79 & 0.781 & 92.94 & 0.88 & 93.68 & 1.21 & 112.3 & 18.7 & 17.3 \\
\hline z5 & 1.2 & 503.8 & 6.9 & 0.169 & 6.7 & 1.2 & 466 & 0.054 & 0.014444 & 0.40 & 0.09574 & 1.06 & 0.04807 & 0.93 & 0.490 & 92.45 & 0.37 & 92.84 & 1.01 & 102.9 & 22.0 & 10.1 \\
\hline \multicolumn{23}{|c|}{ JR1:TONALITE } \\
\hline $\mathrm{z} 1$ & 15.9 & 463.4 & 6.6 & 0.245 & 8.0 & 13 & 538 & 0.078 & 0.014763 & 0.36 & 0.09770 & 0.45 & 0.04800 & 0.27 & 0.808 & 94.47 & 0.33 & 94.65 & 0.43 & 99.2 & 6.3 & 4.8 \\
\hline z2 & 5.2 & 688.7 & 9.8 & 0.261 & 9.1 & 5.6 & 609 & 0.084 & 0.014633 & 0.31 & 0.09725 & 0.47 & 0.04820 & 0.33 & 0.706 & 93.65 & 0.29 & 94.23 & 0.45 & 109.0 & 7.9 & 14.1 \\
\hline z3 & 3.8 & 412.8 & 6.0 & 0.277 & 8.3 & 2.7 & 555 & 0.089 & 0.014816 & 0.34 & 0.09852 & 0.68 & 0.04823 & 0.55 & 0.575 & 94.81 & 0.32 & 95.41 & 0.65 & 110.5 & 13.1 & 14.2 \\
\hline $\mathrm{z} 4$ & 5.4 & 169.7 & 2.5 & 0.302 & 4.3 & 3.1 & 293 & 0.097 & 0.014861 & 0.71 & 0.09858 & 1.36 & 0.04811 & 1.11 & 0.584 & 95.09 & 0.67 & 95.46 & 1.30 & 104.6 & 26.2 & 9.1 \\
\hline z7 & 4.4 & 377.4 & 5.4 & 0.252 & 7.8 & 3.1 & 522 & 0.081 & 0.014803 & 0.36 & 0.09801 & 0.53 & 0.04802 & 0.37 & 0.719 & 94.73 & 0.34 & 94.93 & 0.51 & 100.2 & 8.8 & 5.4 \\
\hline z9 & 0.9 & 862.9 & 12.4 & 0.240 & 10.2 & 1.1 & 681 & 0.077 & 0.014810 & 0.28 & 0.09811 & 0.54 & 0.04805 & 0.44 & 0.577 & 94.77 & 0.26 & 95.03 & 0.51 & 101.5 & 10.4 & 6.6 \\
\hline \multicolumn{23}{|c|}{ PCF:TONALITE } \\
\hline $\mathrm{z} 2$ & 9.4 & 187.0 & 2.7 & 0.323 & 5.4 & 4.8 & 360 & 0.104 & 0.014746 & 0.55 & 0.09791 & 1.47 & 0.04816 & 1.30 & 0.486 & 94.37 & 0.52 & 94.85 & 1.40 & 107.0 & 30.6 & 11.8 \\
\hline z3 & 8.3 & 235.2 & 3.4 & 0.252 & 8.4 & 3.3 & 563 & 0.081 & 0.014748 & 0.35 & 0.09771 & 0.52 & 0.04805 & 0.36 & 0.710 & 94.38 & 0.33 & 94.66 & 0.49 & 101.8 & 8.6 & 7.3 \\
\hline $\mathrm{z} 4$ & 7.4 & 200.9 & 2.9 & 0.321 & 3.3 & 6.7 & 226 & 0.104 & 0.014812 & 0.89 & 0.09882 & 1.39 & 0.04837 & 1.02 & 0.680 & 94.78 & 0.84 & 95.69 & 1.26 & 117.5 & 24.0 & 19.3 \\
\hline z5 & 24.5 & 135.8 & 2.0 & 0.347 & 4.7 & 10 & 314 & 0.112 & 0.014732 & 0.63 & 0.09796 & 0.97 & 0.04823 & 0.71 & 0.688 & 94.28 & 0.59 & 94.89 & 0.93 & 110.4 & 16.7 & 14.6 \\
\hline z6 & 15.4 & 193.3 & 2.9 & 0.431 & 14.6 & 3.0 & 923 & 0.138 & 0.014716 & 0.29 & 0.09758 & 0.53 & 0.04809 & 0.42 & 0.606 & 94.17 & 0.27 & 94.54 & 0.51 & 103.8 & 10.0 & 9.3 \\
\hline z8 & 18.1 & 94.7 & 1.4 & 0.236 & 10.2 & 2.4 & 682 & 0.075 & 0.014831 & 0.28 & 0.09765 & 0.37 & 0.04775 & 0.23 & 0.776 & 94.90 & 0.26 & 94.60 & 0.35 & 87.0 & 5.5 & -9.1 \\
\hline z9 & 14.8 & 155.5 & 2.2 & 0.230 & 11.6 & 2.8 & 780 & 0.074 & 0.014801 & 0.24 & 0.09796 & 0.33 & 0.04800 & 0.22 & 0.755 & 94.72 & 0.23 & 94.89 & 0.32 & 99.3 & 5.2 & 4.6 \\
\hline $\mathrm{z} 10$ & 38.5 & 104.5 & 1.4 & 0.109 & 24.4 & 2.2 & 1672 & 0.035 & 0.014290 & 0.12 & 0.09450 & 0.18 & 0.04797 & 0.13 & 0.693 & 91.47 & 0.11 & 91.69 & 0.17 & 97.6 & 3.1 & 6.2 \\
\hline z11 & 14.7 & 85.8 & 1.2 & 0.094 & 9.1 & 1.9 & 640 & 0.030 & 0.014711 & 0.29 & 0.09752 & 0.46 & 0.04808 & 0.34 & 0.673 & 94.14 & 0.28 & 94.48 & 0.44 & 103.0 & 8.0 & 8.6 \\
\hline $\mathrm{z} 12$ & 7.5 & 80.0 & 1.1 & 0.142 & 4.4 & 1.9 & 316 & 0.046 & 0.014743 & 0.60 & 0.09809 & 0.85 & 0.04826 & 0.57 & 0.740 & 94.35 & 0.56 & 95.01 & 0.81 & 111.8 & 13.5 & 15.6 \\
\hline
\end{tabular}

(b) Sample weights were estimated to within $40 \%$ using measured grain dimensions and a nominal density of $4.5 \mathrm{~g} / \mathrm{cm} 3$ for zircon.

(c) Model $\mathrm{Th} / \mathrm{U}$ ratio calculated from radiogenic ${ }^{208} \mathrm{~Pb} /{ }^{206} \mathrm{~Pb}$ ratio and the ${ }^{207} \mathrm{~Pb} /{ }^{206} \mathrm{~Pb}$ date of the analysis assuming concordance between U-Th- $\mathrm{Pb}$ systems.

(d) $\mathrm{Pb} *$ and $\mathrm{Pbc}$ represent radiogenic $\mathrm{Pb}$ and common $\mathrm{Pb}$ respectively.

(e) Measured ratio corrected for fractionation and spike; Pb fractionation was $0.07 \pm 0.04 \% / a$.m.u. for Faraday detector or $0.25 \pm 0.04 \% / a . m . u$.for Daly detector analysis, based on daily analysis of NBS-981.

(f) Measured ratios corrected for fractionation, spike, and blank; nominal U blank $=0.1 \mathrm{pg} \pm 50 \%$ (2 sigma); all common Pb attributed to blank; measured laboratory Pb

composition: ${ }^{206} \mathrm{~Pb} /{ }^{204} \mathrm{~Pb}=18.27 \pm 0.05,{ }^{207} \mathrm{~Pb} /{ }^{204} \mathrm{~Pb}=15.59 \pm 0.05,{ }^{208} \mathrm{~Pb} /{ }^{204} \mathrm{~Pb}=38.12 \pm 0.05$ (2 sigma). Ratios involving ${ }^{206} \mathrm{~Pb}$ are also corrected for initial disequilibrium in

${ }^{230} \mathrm{Th} /{ }^{238} \mathrm{U}$ assuming a $\mathrm{Th} / \mathrm{U}=4$ in coexisting melt.

(g) \% errors reported at the 2 sigma confidence interval, propagated using the algorithms of Ludwig (1980).

(h) Isotopic ages calculated using the decay constants of Jaffey et al. (1971).

(i) Absolute errors are 2 sigma.

(j) \% discordance $=100-\left[100 \mathrm{x}\left({ }^{206} \mathrm{~Pb} /{ }^{238} \mathrm{U}\right.\right.$ date $) /\left({ }^{207} \mathrm{~Pb} /{ }^{206} \mathrm{~Pb}\right.$ date $\left.)\right]$. 
Table DR2. U-Pb analyses from the Tenpeak pluton.

\begin{tabular}{|c|c|c|c|c|c|c|c|c|c|c|c|c|c|c|c|c|c|c|c|c|c|c|}
\hline \multirow[b]{2}{*}{$\begin{array}{l}\text { Frac } \\
\text { (a) }\end{array}$} & \multicolumn{6}{|c|}{ Composition } & \multicolumn{8}{|c|}{ Isotopic Ratios } & \multicolumn{7}{|c|}{ Dates (Ma) } & \multirow[b]{2}{*}{$\begin{array}{l}\text { Discord- } \\
\text { ance } \\
\text { (j) } \\
\end{array}$} \\
\hline & $\begin{array}{c}\text { Wt } \\
(\mu \mathrm{g}) \\
(\mathrm{b}) \\
\end{array}$ & $\begin{array}{c}\mathrm{U} \\
(\mathrm{ppm})\end{array}$ & $\begin{array}{c}\mathrm{Pb} \\
(\mathrm{ppm})\end{array}$ & $\begin{array}{l}\frac{T h}{U} \\
\text { (c) } \\
\end{array}$ & 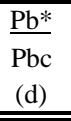 & $\begin{array}{l}\mathrm{Pbc} \\
\text { (pg) } \\
\text { (d) } \\
\end{array}$ & $\begin{array}{c}\frac{{ }^{206} \mathrm{~Pb}}{{ }^{204} \mathrm{~Pb}} \\
(\mathrm{e})\end{array}$ & $\begin{array}{c}\frac{{ }^{208} \mathrm{~Pb}}{{ }^{206} \mathrm{~Pb}} \\
\text { (f) }\end{array}$ & $\begin{array}{c}\frac{{ }^{206} \mathrm{~Pb}}{{ }^{238} \mathrm{U}} \\
\text { (f) }\end{array}$ & $\begin{array}{c}\% \text { err } \\
\text { (g) }\end{array}$ & $\begin{array}{c}\frac{{ }^{207} \mathrm{~Pb}}{{ }^{235} \mathrm{U}} \\
\text { (f) }\end{array}$ & $\begin{array}{c}\% \text { err } \\
\text { (g) }\end{array}$ & $\begin{array}{c}\frac{{ }^{207} \mathrm{~Pb}}{206} \mathrm{~Pb} \\
\text { (f) } \\
\end{array}$ & $\begin{array}{c}\% \text { err } \\
\text { (g) }\end{array}$ & $\begin{array}{l}\text { corr. } \\
\text { coef. }\end{array}$ & $\begin{array}{l}\frac{{ }^{206} \mathrm{~Pb}}{{ }^{238} \mathrm{U}} \\
\text { (h) }\end{array}$ & $\begin{array}{c} \pm \\
\text { (i) }\end{array}$ & $\begin{array}{l}\frac{{ }^{20 /} \mathrm{Pb}}{{ }^{235} \mathrm{U}} \\
\text { (h) } \\
\end{array}$ & $\begin{array}{l} \pm \\
\text { (i) } \\
\end{array}$ & $\begin{array}{c}\frac{{ }^{207} \mathrm{~Pb}}{206} \mathrm{~Pb} \\
(\mathrm{~h}) \\
\end{array}$ & $\begin{array}{l} \pm \\
\text { (i) }\end{array}$ & \\
\hline \multicolumn{23}{|c|}{ "TP1:TONALITE/SHEETED ZONE } \\
\hline $\mathrm{z} 1$ & 9.4 & 220.0 & 3.2 & 0.380 & 20.4 & 1.5 & 1296 & 0.122 & 0.014349 & 0.15 & 0.09468 & 0.22 & 0.04786 & 0.16 & 0.705 & 91.84 & 0.14 & 91.86 & 0.20 & 92.3 & 3.8 & 0.5 \\
\hline z2 & 2.9 & 451.0 & 6.4 & 0.305 & 13.7 & 1.3 & 895 & 0.098 & 0.014361 & 0.22 & 0.09494 & 0.40 & 0.04795 & 0.32 & 0.595 & 91.92 & 0.20 & 92.09 & 0.35 & 96.6 & 7.7 & 4.8 \\
\hline $\mathrm{z} 8$ & 3.3 & 319.4 & 4.6 & 0.343 & 10.2 & 1.5 & 663 & 0.109 & 0.014378 & 0.32 & 0.09466 & 0.79 & 0.04775 & 0.69 & 0.498 & 92.02 & 0.29 & 91.84 & 0.70 & 87.0 & 16.4 & -5.8 \\
\hline $\mathrm{z} 10$ & 9.9 & 94.1 & 1.3 & 0.214 & 14.3 & 0.9 & 958 & 0.069 & 0.014335 & 0.20 & 0.09472 & 0.36 & 0.04792 & 0.29 & 0.601 & 91.76 & 0.18 & 91.89 & 0.32 & 95.4 & 6.9 & 3.8 \\
\hline z11 & 10.6 & 77.3 & 1.1 & 0.341 & 17.4 & 0.7 & 1124 & 0.109 & 0.014326 & 0.18 & 0.09473 & 0.39 & 0.04796 & 0.33 & 0.508 & 91.69 & 0.16 & 91.90 & 0.34 & 97.3 & 7.9 & 5.8 \\
\hline $\mathrm{z} 12$ & 6.1 & 68.6 & 1.0 & 0.431 & 8.3 & 0.7 & 530 & 0.138 & 0.014371 & 0.36 & 0.09523 & 0.64 & 0.04806 & 0.51 & 0.613 & 91.98 & 0.33 & 92.37 & 0.57 & 102.4 & 12.1 & 10.1 \\
\hline \multicolumn{23}{|c|}{ TP11: TONALITE } \\
\hline $\mathrm{z} 1$ & 15.3 & 145.2 & 2.0 & 0.156 & 15.3 & 2.0 & 1042 & 0.050 & 0.014420 & 0.18 & 0.09533 & 0.24 & 0.04795 & 0.15 & 0.777 & 92.29 & 0.17 & 92.46 & 0.21 & 96.7 & 3.6 & 4.6 \\
\hline $\mathrm{z} 4$ & 17.0 & 195.7 & 2.7 & 0.218 & 43.6 & 1.1 & 2884 & 0.070 & 0.014425 & 0.08 & 0.09522 & 0.11 & 0.04788 & 0.08 & 0.680 & 92.32 & 0.07 & 92.36 & 0.10 & 93.2 & 2.0 & 0.9 \\
\hline z5 & 3.8 & 413.5 & 5.6 & 0.120 & 14.5 & 1.5 & 1001 & 0.038 & 0.014409 & 0.19 & 0.09523 & 0.29 & 0.04793 & 0.21 & 0.692 & 92.22 & 0.18 & 92.36 & 0.26 & 95.9 & 5.0 & 3.8 \\
\hline z6 & 17.6 & 62.9 & 0.8 & 0.069 & 25.5 & 0.6 & 1765 & 0.022 & 0.014398 & 0.11 & 0.09506 & 0.20 & 0.04789 & 0.16 & 0.584 & 92.15 & 0.10 & 92.21 & 0.17 & 93.7 & 3.8 & 1.7 \\
\hline z7 & 2.6 & 48.9 & 0.7 & 0.217 & 2.8 & 0.6 & 202 & 0.070 & 0.014493 & 0.93 & 0.09691 & 1.82 & 0.04849 & 1.49 & 0.578 & 92.76 & 0.86 & 93.92 & 1.63 & 123.4 & 35.1 & 24.9 \\
\hline z8 & 3.2 & 70.9 & 1.0 & 0.095 & 4.8 & 0.6 & 344 & 0.031 & 0.014446 & 0.55 & 0.09603 & 1.42 & 0.04821 & 1.24 & 0.498 & 92.46 & 0.51 & 93.11 & 1.26 & 109.7 & 29.3 & 15.7 \\
\hline \multicolumn{23}{|c|}{ TP20: TONALITE } \\
\hline $\mathrm{z} 1$ & 15.6 & 348.7 & 4.8 & 0.242 & 46.1 & 1.6 & 3020 & 0.077 & 0.014377 & 0.08 & 0.09490 & 0.12 & 0.04787 & 0.09 & 0.666 & 92.02 & 0.07 & 92.06 & 0.11 & 93.0 & 2.1 & 1.1 \\
\hline z2 & 11.7 & 572.7 & 8.2 & 0.347 & 101 & 1.0 & 6398 & 0.111 & 0.014380 & 0.06 & 0.09496 & 0.10 & 0.04789 & 0.08 & 0.631 & 92.04 & 0.05 & 92.11 & 0.09 & 93.9 & 1.8 & 2.0 \\
\hline z3 & 10.8 & 311.1 & 4.4 & 0.264 & 48.8 & 1.0 & 3178 & 0.084 & 0.014393 & 0.09 & 0.09501 & 0.16 & 0.04788 & 0.12 & 0.616 & 92.12 & 0.08 & 92.16 & 0.14 & 93.1 & 2.9 & 1.0 \\
\hline $\mathrm{z} 4$ & 7.4 & 290.0 & 4.1 & 0.315 & 36.3 & 0.8 & 2335 & 0.101 & 0.014407 & 0.09 & 0.09525 & 0.14 & 0.04795 & 0.10 & 0.678 & 92.21 & 0.09 & 92.38 & 0.13 & 96.8 & 2.5 & 4.7 \\
\hline z5 & 5.7 & 843.4 & 11.7 & 0.228 & 75.0 & 0.9 & 4924 & 0.073 & 0.014392 & 0.06 & 0.09495 & 0.09 & 0.04785 & 0.07 & 0.673 & 92.12 & 0.06 & 92.10 & 0.08 & 91.7 & 1.7 & -0.5 \\
\hline z6 & 8.7 & 229.8 & 3.2 & 0.237 & 22.7 & 1.2 & 1503 & 0.076 & 0.014384 & 0.14 & 0.09501 & 0.23 & 0.04791 & 0.18 & 0.648 & 92.06 & 0.13 & 92.16 & 0.21 & 94.6 & 4.2 & 2.7 \\
\hline z7 & 6.9 & 389.8 & 5.4 & 0.240 & 41.9 & 0.9 & 2750 & 0.077 & 0.014388 & 0.10 & 0.09492 & 0.20 & 0.04785 & 0.16 & 0.553 & 92.09 & 0.09 & 92.08 & 0.17 & 91.7 & 3.9 & -0.4 \\
\hline z11 & 2.0 & 496.2 & 7.0 & 0.276 & 12.6 & 1.1 & 829 & 0.089 & 0.014396 & 0.24 & 0.09514 & 0.50 & 0.04793 & 0.42 & 0.548 & 92.14 & 0.22 & 92.28 & 0.44 & 95.8 & 9.9 & 3.8 \\
\hline \multicolumn{23}{|c|}{ TP27: TONALITE } \\
\hline $\mathrm{z} 1$ & 15.6 & 116.9 & 1.6 & 0.216 & 19.0 & 1.3 & 1267 & 0.069 & 0.014092 & 0.16 & 0.09343 & 0.27 & 0.04809 & 0.22 & 0.603 & 90.21 & 0.14 & 90.70 & 0.24 & 103.6 & 5.2 & 12.9 \\
\hline z2 & 10.2 & 122.0 & 1.7 & 0.259 & 17.7 & 1.0 & 1164 & 0.083 & 0.014160 & 0.17 & 0.09352 & 0.41 & 0.04790 & 0.35 & 0.508 & 90.64 & 0.16 & 90.77 & 0.35 & 94.3 & 8.3 & 3.8 \\
\hline z3 & 12.2 & 250.8 & 3.4 & 0.161 & 49.9 & 0.8 & 3352 & 0.051 & 0.014151 & 0.10 & 0.09338 & 0.17 & 0.04786 & 0.14 & 0.602 & 90.58 & 0.09 & 90.65 & 0.15 & 92.3 & 3.2 & 1.9 \\
\hline $\mathrm{z} 4$ & 21.5 & 101.5 & 1.4 & 0.195 & 11.5 & 2.6 & 781 & 0.062 & 0.014188 & 0.24 & 0.09378 & 0.35 & 0.04794 & 0.24 & 0.732 & 90.82 & 0.22 & 91.02 & 0.30 & 96.4 & 5.6 & 5.8 \\
\hline z5 & 19.2 & 71.2 & 1.0 & 0.286 & 17.8 & 1.1 & 1163 & 0.092 & 0.014167 & 0.16 & 0.09359 & 0.29 & 0.04792 & 0.23 & 0.616 & 90.68 & 0.15 & 90.84 & 0.25 & 95.1 & 5.4 & 4.6 \\
\hline z6 & 14.8 & 181.1 & 2.4 & 0.157 & 37.6 & 1.0 & 2532 & 0.050 & 0.014163 & 0.09 & 0.09345 & 0.17 & 0.04785 & 0.14 & 0.560 & 90.66 & 0.08 & 90.71 & 0.15 & 92.0 & 3.4 & 1.5 \\
\hline z7 & 9.5 & 555.9 & 7.3 & 0.084 & 54.8 & 1.3 & 3763 & 0.027 & 0.014149 & 0.07 & 0.09333 & 0.11 & 0.04784 & 0.08 & 0.657 & 90.57 & 0.06 & 90.60 & 0.09 & 91.3 & 1.9 & 0.8 \\
\hline \multicolumn{23}{|c|}{ TP29: FLASER GNEISS } \\
\hline $\mathrm{z} 1$ & 8.1 & 401.9 & 5.4 & 0.141 & 15.4 & 2.8 & 1052 & 0.045 & 0.014256 & 0.18 & 0.09413 & 0.25 & 0.04789 & 0.16 & 0.765 & 91.25 & 0.17 & 91.35 & 0.22 & 93.8 & 3.8 & 2.7 \\
\hline z2 & 9.5 & 213.0 & 2.9 & 0.177 & 12.7 & 2.2 & 863 & 0.057 & 0.014278 & 0.22 & 0.09431 & 0.30 & 0.04791 & 0.19 & 0.764 & 91.39 & 0.20 & 91.51 & 0.26 & 94.7 & 4.5 & 3.5 \\
\hline z3 & 4.2 & 499.5 & 6.7 & 0.157 & 7.3 & 3.8 & 508 & 0.050 & 0.014276 & 0.38 & 0.09440 & 0.57 & 0.04796 & 0.41 & 0.694 & 91.38 & 0.34 & 91.59 & 0.50 & 97.2 & 9.8 & 6.0 \\
\hline $\mathrm{z} 4$ & 3.5 & 422.6 & 5.7 & 0.188 & 4.7 & 4.3 & 331 & 0.060 & 0.014286 & 0.58 & 0.09457 & 0.74 & 0.04801 & 0.44 & 0.806 & 91.44 & 0.53 & 91.75 & 0.65 & 99.8 & 10.4 & 8.4 \\
\hline z6 & 4.4 & 464.9 & 6.2 & 0.141 & 15.2 & 1.8 & 1040 & 0.045 & 0.014252 & 0.18 & 0.09405 & 0.25 & 0.04786 & 0.16 & 0.754 & 91.23 & 0.16 & 91.27 & 0.22 & 92.5 & 3.9 & 1.4 \\
\hline z7 & 1.5 & 1672 & 22.2 & 0.114 & 10.8 & 3.0 & 751 & 0.037 & 0.014253 & 0.26 & 0.09423 & 0.38 & 0.04795 & 0.26 & 0.709 & 91.23 & 0.23 & 91.44 & 0.33 & 96.9 & 6.3 & 5.9 \\
\hline
\end{tabular}


Table DR2 continued. U-Pb analyses from the Tenpeak pluton.

\begin{tabular}{|c|c|c|c|c|c|c|c|c|c|c|c|c|c|c|c|c|c|c|c|c|c|c|}
\hline \multirow[b]{2}{*}{$\begin{array}{c}\text { Frac } \\
\text { (a) }\end{array}$} & \multicolumn{6}{|c|}{ Composition } & \multicolumn{9}{|c|}{ Isotopic Ratios } & \multicolumn{6}{|c|}{ Dates (Ma) } & \multirow[b]{2}{*}{$\begin{array}{c}\text { Discord- } \\
\text { ance } \\
\text { (j) } \\
\end{array}$} \\
\hline & $\begin{array}{c}\text { Wt } \\
(\mu \mathrm{g}) \\
(\mathrm{b})\end{array}$ & $\begin{array}{c}\mathrm{U} \\
(\mathrm{ppm})\end{array}$ & $\begin{array}{c}\mathrm{Pb} \\
(\mathrm{ppm})\end{array}$ & $\begin{array}{l}\frac{\mathrm{Th}}{\mathrm{U}} \\
\text { (c) } \\
\end{array}$ & $\begin{array}{l}\frac{\mathrm{Pb}^{*}}{\mathrm{Pbc}} \\
\text { (d) }\end{array}$ & $\begin{array}{l}\mathrm{Pbc} \\
\text { (pg) } \\
\text { (d) } \\
\end{array}$ & $\begin{array}{c}\frac{{ }^{206} \mathrm{~Pb}}{{ }^{204} \mathrm{~Pb}} \\
\text { (e) }\end{array}$ & $\begin{array}{l}\frac{{ }^{208} \mathrm{~Pb}}{{ }^{206} \mathrm{~Pb}} \\
\text { (f) }\end{array}$ & $\begin{array}{c}\frac{{ }^{206} \mathrm{~Pb}}{{ }^{238} \mathrm{U}} \\
\text { (f) }\end{array}$ & $\begin{array}{l}\text { \% err } \\
\text { (g) }\end{array}$ & $\begin{array}{c}\frac{{ }^{207} \mathrm{~Pb}}{{ }^{235} \mathrm{U}} \\
\text { (f) }\end{array}$ & $\begin{array}{c}\% \text { err } \\
\text { (g) }\end{array}$ & $\begin{array}{c}\frac{{ }^{207} \mathrm{~Pb}}{{ }^{206} \mathrm{~Pb}} \\
\text { (f) }\end{array}$ & $\begin{array}{c}\% \text { err } \\
\text { (g) }\end{array}$ & $\begin{array}{l}\text { corr. } \\
\text { coef. }\end{array}$ & $\begin{array}{c}\frac{{ }^{206} \mathrm{~Pb}}{{ }^{238} \mathrm{U}} \\
\text { (h) }\end{array}$ & $\begin{array}{c} \pm \\
\text { (i) }\end{array}$ & $\begin{array}{l}\frac{{ }^{200} \mathrm{~Pb}}{{ }^{235} \mathrm{U}} \\
\text { (h) }\end{array}$ & $\begin{array}{l} \pm \\
\text { (i) }\end{array}$ & $\begin{array}{c}\frac{207 \mathrm{~Pb}}{{ }^{206} \mathrm{~Pb}} \\
\text { (h) }\end{array}$ & $\begin{array}{l} \pm \\
\text { (i) }\end{array}$ & \\
\hline \multicolumn{23}{|c|}{ TP30: DIORITE IN MAFIC COMPLEX } \\
\hline $\mathrm{z} 1$ & 8.9 & 294.0 & 4.1 & 0.266 & 7.3 & 5.0 & 491 & 0.085 & 0.014380 & 0.39 & 0.09506 & 0.60 & 0.04794 & 0.43 & 0.692 & 92.04 & 0.36 & 92.21 & 0.53 & 96.5 & 10.3 & 4.6 \\
\hline $\mathrm{z} 2$ & 3.5 & 294.3 & 4.1 & 0.241 & 7.3 & 1.9 & 497 & 0.077 & 0.014395 & 0.38 & 0.09526 & 0.56 & 0.04800 & 0.39 & 0.712 & 92.13 & 0.35 & 92.39 & 0.49 & 99.1 & 9.3 & 7.0 \\
\hline z6 & 3.2 & 181.9 & 2.5 & 0.162 & 6.0 & 1.3 & 417 & 0.052 & 0.014389 & 0.45 & 0.09561 & 0.74 & 0.04819 & 0.56 & 0.659 & 92.10 & 0.41 & 92.71 & 0.66 & 108.6 & 13.2 & 15.2 \\
\hline z7 & 2.4 & 165.0 & 2.3 & 0.237 & 4.9 & 1.1 & 339 & 0.076 & 0.014422 & 0.58 & 0.09575 & 1.33 & 0.04815 & 1.14 & 0.524 & 92.30 & 0.53 & 92.85 & 1.18 & 106.8 & 26.8 & 13.6 \\
\hline z8 & 1.7 & 283.3 & 4.1 & 0.303 & 6.9 & 1.0 & 460 & 0.098 & 0.014443 & 0.41 & 0.09577 & 0.88 & 0.04809 & 0.75 & 0.531 & 92.44 & 0.37 & 92.86 & 0.78 & 103.8 & 17.7 & 11.0 \\
\hline z9 & 1.9 & 139.9 & 2.0 & 0.312 & 4.0 & 0.9 & 276 & 0.101 & 0.014468 & 0.68 & 0.09649 & 1.09 & 0.04837 & 0.81 & 0.669 & 92.60 & 0.63 & 93.53 & 0.97 & 117.3 & 19.1 & 21.1 \\
\hline \multicolumn{23}{|c|}{ TP31: TONALITE (WHITE MOUNTAIN LOBE) } \\
\hline $\mathrm{z} 3$ & 2.4 & 120.1 & 1.8 & 0.497 & 2.5 & 1.7 & 171 & 0.161 & 0.014447 & 1.17 & 0.09659 & 1.64 & 0.04849 & 1.10 & 0.743 & 92.46 & 1.07 & 93.62 & 1.47 & 123.3 & 25.9 & 25.0 \\
\hline $\mathrm{z} 4$ & 1.3 & 79.1 & 1.2 & 0.411 & 2.5 & 0.6 & 171 & 0.133 & 0.014477 & 1.15 & 0.09696 & 2.34 & 0.04857 & 1.93 & 0.567 & 92.66 & 1.05 & 93.96 & 2.10 & 127.2 & 45.5 & 27.2 \\
\hline z6 & 1.7 & 99.5 & 1.5 & 0.333 & 3.2 & 0.8 & 221 & 0.109 & 0.014660 & 0.86 & 0.09935 & 1.61 & 0.04915 & 1.30 & 0.587 & 93.82 & 0.80 & 96.18 & 1.47 & 155.1 & 30.5 & 39.5 \\
\hline z9 & 3.0 & 33.6 & 0.5 & 0.401 & 1.8 & 0.8 & 130 & 0.131 & 0.014500 & 1.56 & 0.09762 & 4.54 & 0.04883 & 4.03 & 0.479 & 92.80 & 1.44 & 94.57 & 4.10 & 139.5 & 94.7 & 33.5 \\
\hline \multicolumn{23}{|c|}{ TP-524-1: TONALITE } \\
\hline $\mathrm{z} 1$ & 11.3 & 130.3 & 1.8 & 0.237 & 20.7 & 1.0 & 1370 & 0.076 & 0.014002 & 0.15 & 0.09241 & 0.28 & 0.04786 & 0.24 & 0.555 & 89.63 & 0.13 & 89.74 & 0.24 & 92.6 & 5.6 & 3.2 \\
\hline $\mathrm{z} 2$ & 6.8 & 108.8 & 1.5 & 0.274 & 14.8 & 0.7 & 977 & 0.088 & 0.013993 & 0.21 & 0.09240 & 0.40 & 0.04789 & 0.32 & 0.587 & 89.58 & 0.19 & 89.73 & 0.34 & 93.9 & 7.6 & 4.6 \\
\hline z7 & 6.5 & 77.1 & 1.1 & 0.264 & 6.4 & 1.1 & 431 & 0.085 & 0.014037 & 0.44 & 0.09307 & 0.68 & 0.04809 & 0.50 & 0.686 & 89.86 & 0.39 & 90.36 & 0.59 & 103.7 & 11.7 & 13.3 \\
\hline $\mathrm{z} 13$ & 9.5 & 95.7 & 1.3 & 0.285 & 20.8 & 0.6 & 1358 & 0.091 & 0.014026 & 0.16 & 0.09247 & 0.39 & 0.04782 & 0.34 & 0.488 & 89.79 & 0.14 & 89.81 & 0.33 & 90.3 & 8.1 & 0.5 \\
\hline z14 & 6.7 & 27.1 & 0.4 & 0.339 & 5.3 & 0.5 & 357 & 0.109 & 0.014021 & 0.54 & 0.09292 & 1.83 & 0.04806 & 1.65 & 0.456 & 89.76 & 0.48 & 90.22 & 1.58 & 102.4 & 39.1 & 12.4 \\
\hline
\end{tabular}

(a) All zircon fractions (i.e. z1) are composed of single grains or fragments.

(b) Sample weights were estimated to within $40 \%$ using measured grain dimensions and a nominal density of $4.5 \mathrm{~g} / \mathrm{cm} 3$ for zircon.

(c) Model $\mathrm{Th} / \mathrm{U}$ ratio calculated from radiogenic ${ }^{208} \mathrm{~Pb} /{ }^{206} \mathrm{~Pb}$ ratio and the ${ }^{207} \mathrm{~Pb} /{ }^{206} \mathrm{~Pb}$ date of the analysis assuming concordance between U-Th- $\mathrm{Pb}$ systems.

(d) $\mathrm{Pb} *$ and $\mathrm{Pbc}$ represent radiogenic $\mathrm{Pb}$ and common $\mathrm{Pb}$ respectively.

(e) Measured ratio corrected for fractionation and spike; Pb fractionation was $0.07 \pm 0.04 \% /$ a.m.u. for Faraday detector or $0.25 \pm 0.04 \% /$ a.m.u.for Daly detector analysis, based on daily analysis of NBS-981.

(f) Measured ratios corrected for fractionation, spike, and blank; nominal U blank $=0.1 \mathrm{pg} \pm 50 \%$ (2 sigma); all common Pb attributed to blank; measured laboratory Pb

composition: ${ }^{206} \mathrm{~Pb} /{ }^{204} \mathrm{~Pb}=18.27 \pm 0.05,{ }^{207} \mathrm{~Pb} /{ }^{204} \mathrm{~Pb}=15.59 \pm 0.05,{ }^{208} \mathrm{~Pb} /{ }^{204} \mathrm{~Pb}=38.12 \pm 0.05$ (2 sigma). Ratios involving ${ }^{206} \mathrm{~Pb}$ are also corrected for initial disequilibrium in ${ }^{230} \mathrm{Th} /{ }^{238} \mathrm{U}$ assuming a $\mathrm{Th} / \mathrm{U}=4$ in coexisting melt.

(g) \% errors reported at the 2 sigma confidence interval, propagated using the algorithms of Ludwig (1980).

(h) Isotopic ages calculated using the decay constants of Jaffey et al. (1971).

(i) Absolute errors are 2 sigma.

(j) \% discordance $=100-\left[100 \times{ }^{206} \mathrm{~Pb} /{ }^{238} \mathrm{U}\right.$ date $) /\left({ }^{207} \mathrm{~Pb} /{ }^{206} \mathrm{~Pb}\right.$ date $\left.)\right]$. 